\title{
Temperature-insensitive fiber optic deformation sensor embedded in composite material
}

\author{
A. W. Domański ${ }^{1}$, P. Lesiak ${ }^{1}$, K. Milenko ${ }^{1}$, A. Boczkowska ${ }^{2}$, D. Budaszewski ${ }^{1}$, S. Ertman ${ }^{1}$, and T. R. Woliński ${ }^{1}$, \\ ${ }^{1}$ Faculty of Physics, Warsaw University of Technology, Koszykowa 75, 00-662 Warszawa, \\ ${ }^{2}$ Faculty of Materials Science and Engineering, Warsaw University of Technology, Wołoska 141, 02-507 Warsaw,
}

Received September 29, 2009; accepted September 30, 2009; published September 30, 2009

\begin{abstract}
Composite structures are made of two or more components with significantly different physical or chemical properties and they remain separate and distinct in a macroscopic level within the finished structure. This feature allows for introducing optical fiber sensors into the composite material. These sensors can demonstrate stress distribution inside tested material influenced by external tensions. In the function of the sample bend the biggest deformation is created at the surface. In the middle of the sample strain is almost equal to zero. A fiber optic sensor placed in the center of the composite material is sensitive only to temperature changes, whereas a fiber sensor placed on the surface is sensitive simultaneously to temperature and to deformations. The use of the centre fiber as a temperature compensation component is presented in this paper.
\end{abstract}

Polarimetric optical fiber sensors based on highly birefringent $(\mathrm{HB})$ polarization-maintaining fibers have focused great interest for the last decades [1, 2]. In HB fibers, the difference between the phase velocities for the two orthogonally polarized modes is high enough to avoid coupling between these two modes. Fibers of this class have a built-in, well-defined, high internal birefringence obtained by designing a core and/or cladding with noncircular (mostly elliptical) geometry, or by using anisotropic stress applying parts built into the cross-section of the fiber.

The modal behavior of the lowest-order mode HB fibers under various external deformations is of special interest for sensors and device applications. A number of physical quantities can be measured on the basis of HB fibers: hydrostatic pressure, strain, vibration, temperature, acoustic wave, etc.

A symmetric deformation effect (X) influences the propagation constant $\beta$ in every mode because of the changes in fiber length $L$ and the refractive indices of the core and the cladding. In a single-mode regime, this leads to changes in the phase difference between both polarizations of the fundamental LP01 mode along the fiber [1]:

$$
\frac{\delta(\Delta \Phi)}{\delta X}=\Delta \beta \frac{\partial L}{\partial X}+L \frac{\partial(\Delta \beta)}{\partial X}
$$

where $X$ stands for temperature $(T)$, pressure $(\mathrm{p})$ or longitudinal strain $(\varepsilon)$ defined as: $\varepsilon=\Delta L / L$.
The effect of longitudinal strain on mode coupling is to modulate the relative phase retardation between the two orthogonal polarizations in the $\mathrm{LP}_{01}$ mode. The general formula describing birefringence sensitivity to strain can be expressed in terms of an experimental parameter $T_{\varepsilon}$ describing the amount of strain $\varepsilon$ required to induce a $2 \pi$ phase shift of polarized light observed at the output as [3]:

$$
\Delta \beta(\varepsilon)=\Delta \beta^{0}+\operatorname{sgn}\left[\frac{d(\Delta \beta)}{d \varepsilon}\right] \varepsilon \frac{2 \pi}{T_{\varepsilon} L}
$$

where $\Delta \beta^{0}$ signifies unperturbated polarization birefringence of the fiber and the function $\operatorname{sgn}[\mathrm{d}(\Delta \beta) / \mathrm{d} \varepsilon]$ has two values: +1 or -1 depending on the sign of the changes in the relative polarization birefringence with strain and $L$ is the total optical path of the fiber.

Under the influence of longitudinal strain the first term on the right-hand side of expression (1) is negligible with respect to the first, so that:

$$
\delta(\Delta \Phi) \cong \frac{\partial(\Delta \beta)}{\partial \varepsilon} L \varepsilon=\frac{\partial(\Delta \beta)}{\partial \varepsilon} \delta L
$$

Hence the phase changes of the polarimetric responses are proportional to absolute elongation $\Delta \mathrm{L}$ and are independent of length $\mathrm{L}$ of the sensing region. Under the influence of longitudinal axial strain, the equation (1) can be approximated with the use of formulae (2) in terms of the only experimental parameter $\mathrm{T}_{\varepsilon}$

$$
\frac{\delta(\Delta \Phi)}{\delta \varepsilon}=\Delta \beta \frac{\partial L}{\partial \varepsilon}+\operatorname{sgn} \frac{d(\Delta \beta)}{d \varepsilon} \cdot \frac{2 \pi}{T_{\varepsilon}} \cong \operatorname{sgn} \frac{d(\Delta \beta)}{d \varepsilon} \cdot \frac{2 \pi}{T_{\varepsilon}}
$$

In recent years, many experiments and researches have been carried out on laminated composites to obtain optimal mechanical properties [4-11]. Composite properties are highest in the direction of fibers orientation. In a practical application, most of the structures are not loaded in one direction, and it is necessary to orient fibers in different directions. This 
demands evaluation of mechanical properties for different fiber orientations. The fiber weight fraction is an important parameter influencing the mechanical properties of composites.

The stress-induced $(\varepsilon)$ elongation of the sample in the function of the deflection is given by the following formula:

$$
\varepsilon=6 \frac{s \cdot d}{L^{2}}
$$

where: s - deflection, d - distance between fiber layers, $\mathrm{L}$ - length of the sample.

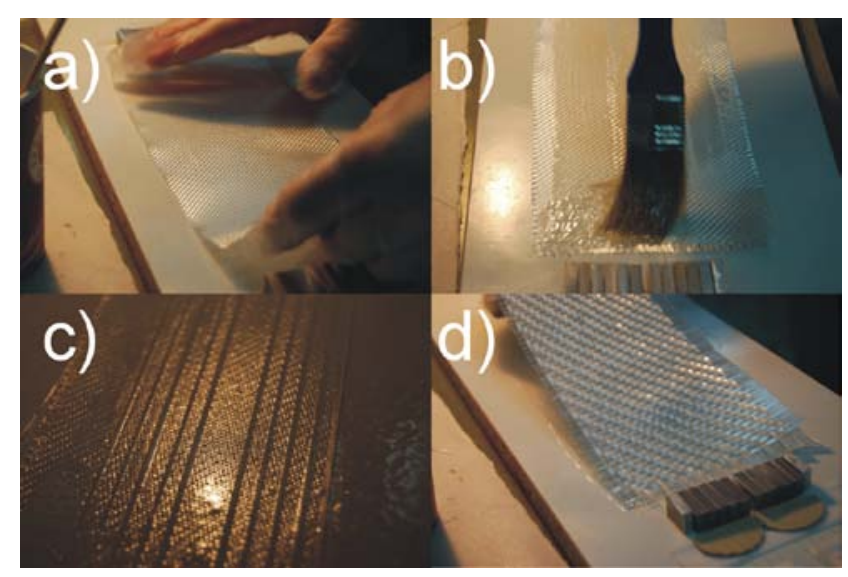

Fig. 1. Sample preparation process: a) first 2D fabrics layer; b) epoxy resin; c) fiber optic sensors layer; d) next $2 \mathrm{D}$ fabrics layer

The 3-d structure of 15 polarimetric sensors was implemented into the composite materials. The sample was $200 \mathrm{~mm}$ long, $25 \mathrm{~mm}$ wide and $2.5 \mathrm{~mm}$ thick. Additionally, the sample had the same distance between fiber sensors layers $-\mathrm{d}=1 \mathrm{~mm}$. The sample was made at the Faculty of Materials Science and Engineering, Warsaw University of Technology (Fig. 1.).

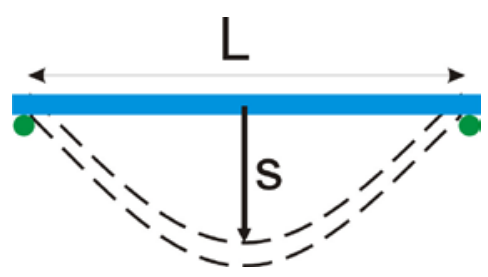

Fig. 2. Scheme of the deflection measurements

The measurement setup consists of a Point-Source laser as the light source: output power $3 \mathrm{~mW}, \lambda=850 \mathrm{~nm}$ and a polarization analyzer. The Peltier thermoelectric device was used for controlled temperature changes.
Measurement results show that stress-induced effects in the fiber optic sensors correspond to stress distribution in the deformed composite material. The position of the sensing fiber (in the middle or above the center of the sample) plays a predominant role in the stress-induced effects. Polarization changes were only observed in the sensor placed above the center of the sample.

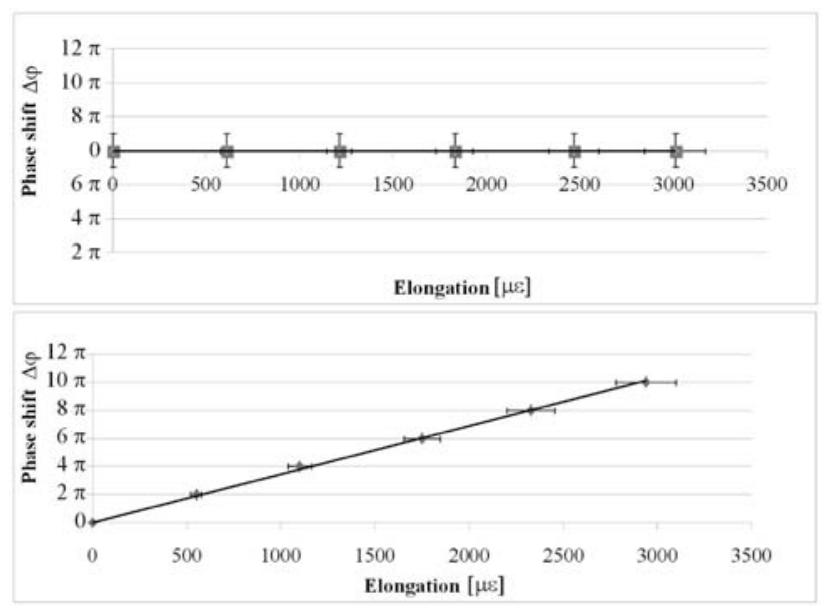

Fig. 3. Change of the polarization in the function of the sample bend for fiber optic sensors placed in the middle (top) and above (bottom) the centre of the sample

Temperature field acting on the HB fiber causes a change in the dimensions of the fiber and in the refractive indices. Hence, any polarimetric strain or a pressure sensor is inevitably temperature-dependent and so ambient thermal fluctuations cause instabilities in the output reading. Thus, temperature compensation becomes of prime importance for the proper work of a polarimetric fiber-optic sensor.

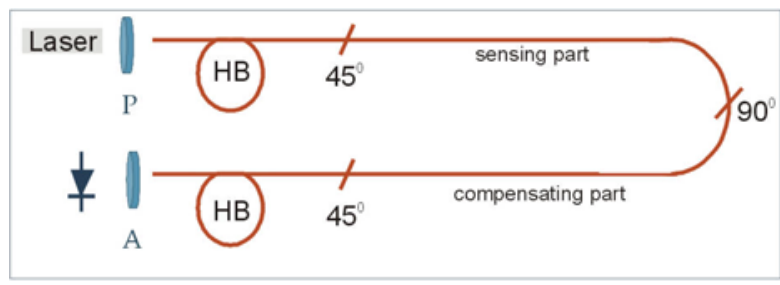

Fig. 4. Fiber optic sensor with temperature compensation part

To reduce the disturbing influence of temperature on the characteristics of an $\mathrm{HB}$ fiber based single-mode polarimetric sensor, a compensation scheme is proposed (Fig. 3.) in which two pieces of the same fiber with identical lengths are spliced with birefringence axes rotated at 90 degrees to each other. The first fiber (placed in the middle of the centre of composite material) is sensitive only to temperature changes. The second one (placed above the middle of the centre of composite material) is sensitive simultaneously to temperature changes and to deformations.. This is a polarization mode 
interchanger in which the fast mode in the first section is coupled to the slow mode of the second section. In this case the strain sensor will be immune to ambient temperature or any other physical parameter acting on both its parts.

The measurements data presented in Fig. 5. demonstrate that the sensor with a temperature compensation part does not change its strain sensitivity for the same elongation of the sample.



Fig. 5. Change of the polarization in the function of temperature for fiber optic sensors with temperature compensation part

The measurement results presented in this paper show that stress-induced effects in the fiber optic sensors correspond to stress distribution in the deformed composite material. Additionally, it is possible to construct in the composite material a fiber optic sensor with zero-temperature sensitivity.

This work was supported by the Polish Ministry of Science and Higher Education through The National Centre for Research and Development under the grant ERA-NET MATERA.

\section{References}

[1] T. R. Wolinski, "Polarimetric Optical Fibers and Sensors”, Progress in Optics, ed. Emil Wolf (North Holland, Amsterdam), vol. XL, pp. 1-75 (2000)

[2] T. R. Wolinski, "Polarization Phenomena in Optical Systems", in Enc. Opt. Engineering, ed. R. Diggers, M. Dekker, New York, pp. 2150-2175 (2003)

[3] W. J. Bock, A. W. Domanski, T. R. Wolinski, "Influence of High Hydrostatic Pressure on Beat Length in Highly Birefringent Single-Mode Bow-Tie Fibers”, Appl. Optics, vol. 29, 3484-3488 (1990)

[4] Kuang KSC, Kenny R, Whelan MP, Cantwell WJ, Chalker PR "Embedded fiber Bragg grating sensors in advanced composite materials”, Composite Science and Technology, 61, (2001) 1379 1387

[5] Guemes JA, Diaz-Carrilo Menendes JM. Measurement of strain distribution in bonded joints by fiber Bragg gratings. SPIE 1998;3330:264-71.

[6] Guemes JA, Diaz-Carrilo Menendes JM. "Response of Bragg grating fiber-optic sensors when embedded in composite laminates”, Composite Science and Technology, 62, (2002) 959966

[7] Reyes G, Cantwell WJ. The mechanical properties of fiber-metal laminates based on glass fibre reinforced polypropylene. Composites Science and Technology 2000;60:1085-94.

[8] Davies P, Cantwell WJ. Fracture of glass/polypropylene laminates: influence of cooling rate after moulding. Composites 1994;25:869-77.
[9] Peters K, Studer M, Botsis J, Iocco A, Limberger HG, Salathe RP. Measurement of stress concentrations using embedded optical fiber Bragg grating sensors. SPIE 1999;3670:195-206.

[10] T. K. Gangopadhyay, M. Majumder, A. K. Chakraborty, A. K. Dikshit, D. K. Bhattacharya, "Fibre Bragg grating strain sensor and study of its packaging material for use in critical analysis on steel structure”, Sensors and Actuators A, 150, (2009), 78-86

[11] N. C. Eaton, M. J. Curran, J. P. Dakin, H. Geiger, "Fiber Optic Bragg Grating Sensor Measurements in Composite Materials", Smart Composites, 20-24 September 1993, Bordeaux, France 